\title{
Nivel de compromiso deportivo en tenistas y futbolistas entre 10 y 14 años
}

\section{Sport commitment level in tennis and soccer players among 10-14 years}

\author{
Paula Ortiz Marholz ${ }^{1} \quad$ Enrique Arriaza Ardiles \\ Vanessa Jeria Santander \\ Universidad de Playa Ancha, Chile \\ (Rec: Octubre 2011 - Acep: Noviembre 2011)
}

\begin{abstract}
Resumen
En el presente estudio se pretende determinar el nivel de compromiso deportivo en un grupo de tenistas y futbolistas, de manera de conocer si esta información puede ayudar a monitorear y controlar el entrenamiento deportivo, desde la perspectiva de la motivación de logro. La muestra está formada por 174 varones con edades comprendidas entre los 10 y 14 años, provenientes de las disciplinas deportivas de tenis $(\mathrm{n}=31)$ y fútbol $(n=143)$. Los resultados mostraron un alto nivel de compromiso de los sujetos hacia su práctica deportiva, lo que se fundamenta en la relación existente entre los valores obtenidos por los distintos factores evaluados. Se concluye que estos deportistas exiben una disposición psicológica positiva que se refleja en el deseo y decisión de seguir participando en su respectiva disciplina deportiva, lo que se presenta como factor protector frente al abandono deportivo.
\end{abstract}

Palabras clave: compromiso deportivo, motivación de logro, deportistas jóvenes.

\begin{abstract}
This study is to determine the level of sport commitment in a group of tennis players and soccer players in order to know if this information can help to monitor and control the sport training from the perspective of achievement motivation. The sample consists of 174 male subjects aged between 10 and 14 years old coming from different sports disciplines such as tennis $\left(n^{\circ}=31\right)$ and soccer $\left(n^{\circ}=143\right)$. The results showed a high level of commitment shown by the subjects toward sport practice which is based on the existing relationship between the values obtained by the different factors evaluated. It was concluded that the athletes evaluated have a positive psychological disposition which is reflected in the desire and decision to continue participating in their respective sport discipline that is presented as a protection factor against the drop-out.
\end{abstract}

Key words: sports commitment, achievement motivation, young athletes.

\footnotetext{
1 Correspondencia a: Paula Ortiz Marholz. Dirección: Facultad de Ciencias de la Actividad Física y el Deporte. Avenida Playa Ancha 850. Valparaíso. Tel: 56 - 032 - 2500115. E-mail: ortiz.m.paula@gmail.com.
} 


\section{Introducción}

El objetivo de este trabajo es determinar el nivel de compromiso deportivo de los alumnos a la luz de la teoría de Compromiso Deportivo (Scanlan, Carpenter, Schmidt, Simons \& Keeler, 1993). Nuestra intención es conocer si esta teoría puede contribuir con información que coadyuve en el monitoreo y control del entrenamiento desde la perspectiva de la motivación de logro.

Se sabe que existen ciertos factores, externos al deportista, que afectan la participación en la actividad y consecuentemente, su proyección deportiva. Estos factores se relacionan con los otros significativos que se asocian a la consecución de su participación. Estos otros significativos, en específico en este caso los padres y el entrenador, tienen conductas que pueden ser facilitadores o perjudiciales a la continuidad en la actividad, así como influir en la motivación y nivel de compromiso.

La teoría define al compromiso deportivo como la disposición psicológica de una persona que representa el deseo y la decisión de mantener su participación en la actividad física y deportiva (Scalan et al., 1993). Se infiere, por tanto, que existen ciertos factores internos y externos al deportista que afectan su participación en la actividad y consecuentemente su proyección deportiva.

Este constructo se aplica para referirse a la motivación por continuar en la práctica deportiva, actuando como un factor protector ante el desgaste en el deporte y ante el abandono (Weiss \& Petlichkoff, 1989), y proporcionando consecuentemente un marco teórico referencial para estudiar los procesos subyacentes. Originalmente el Compromiso ha sido utilizado bajo otros contextos (Kelley \& Thibaut, 1978; Rusbult, 1980; Rusbut \& Farrel, 1983), los que han nutrido la construcción del modelo.

El modelo de Compromiso Deportivo (Scanlan et al., 1993) plantea que los determinantes de éste pueden sintetizarse en los conceptos psicológicos de atracción, alternativas y fuerzas restrictivas. La variable atracción está representada por el disfrute del deporte. El concepto de alternativas se asocia al determinante del modelo de alternativas de implicación. Para las fuerzas restrictivas o barreras, el modelo plantea tres que pudieran afectar al compromiso deportivo: inversiones personales para con la actividad, el impacto de las normas sociales, definida como coacción social, y la promesa que las futuras oportunidades en la actividad se deriven de su participación continua, definida como oportunidades de implicación.
Su estructuración postula cinco factores determinantes del compromiso. El protocolo de aplicación los mide separadamente, lo que permite, por un lado, evaluar y predecir la influencia en el compromiso deportivo independientemente y por otro, intervenir específicamente en aquel que presente dificultad. Estos factores interrelacionados afectan el nivel de compromiso.

El grado de diversión es definido como una respuesta afectiva positiva a la experiencia deportiva, que se refleja en sentimientos generalizados de placer, gusto y diversión ; la inversión personal, que se establece como los recursos personales que son puestos en la actividad y que no pueden ser recuperados si la participación en la actividad culmina; las oportunidades de implicación, concebidas como la valoración de oportunidades que se presentan solo a través de una participación continua; las coacciones sociales, entendidas como las expectativas sociales o normas que crean sentimientos de obligación de continuar en la actividad; y las alternativas de implicación, que se refieren al atractivo de la(s) alternativa(s) preferida(s) para continuar participando y esforzándose (Scanlan et al., 1993). Es así, entonces, que el compromiso deportivo se ve afectado por el aumento de alternativas en las que potencial o efectivamente el deportista se vea involucrado.

En teoría lo esperable es que grado de diversión, oportunidades de implicación e inversiones personales presenten altos valores contribuyendo positivamente al compromiso deportivo, en contraste alternativas de implicación y coacciones sociales debieran mostrar bajos valores, no afectando negativamente el compromiso. De esta manera, se asocia la sensación de satisfacción a la práctica deportiva con la adhesión y permanencia a ella (García, Leo, Martín \& Sánchez, 2008).

Algunas investigaciones han profundizado en estos aspectos; Souza (2008) solo encontró que el grado de diversión afecta positivamente el Compromiso Deportivo, en tanto las alternativas de implicación lo hacen en forma negativa. García Más, Palou, Gili otros (2010), por otra parte, plantean que la definición de disfrute implica una relación causal entre preferencia por la actividad deportiva comparativamente con el peso de la percepción de la alternativa en el deporte; la satisfacción interna de la persona y el sentimiento de competencia mientras realiza el deporte.

Los motivos para la participación en la actividad física y el deporte han sido ampliamente considerados (Cecchini, Méndez \& Muñiz, 2002; Cervelló, Itutzler, Reina, Sanz \& Moreno, 2005), para explicar la importancia de los factores tanto internos como externos que 
pudieran estar afectando al compromiso. Esto tiene relevancia en cuanto poder intervenir en ellos para permitir desarrollar herramientas que ayuden a encontrar soluciones ante el desgaste y evitar así, el abandono deportivo (Hernández-Mendo \& Blanco, 2005).

\section{Método}

Dadas las características de los datos y de los instrumentos de recolección de los mismos, se define como un estudio cuantitativo, descriptivo de diseño no experimental y transversal (Thomas, 2007).

\section{Participantes}

En este estudio participaron 174 sujetos con edades comprendidas entre los 10 y 14 años $(\mathrm{M}=12,2$; DT $=1,3)$, pertenecientes a las modalidades deportivas de tenis $(n=31)$ y fútbol $(n=143)$ de clubes particulares en el primer caso y series cadetes de equipos profesionales, para el segundo. Se consideró como criterio de exclusión la permanencia menor a 3 meses y que no hayan jugado partido alguno con su entrenador actual. La muestra definitiva se compuso de 135 personas.

\section{Instrumentos}

El nivel de compromiso se evaluó a través del SCQ-e (The Sport Commitment Model Questionnaire) de Scalan, 1993. Este instrumento se basa en el Modelo de Compromiso Deportivo propuesto por Scalan (Sport Commitment Model, versión español), el cual mide 6 factores influyentes en el nivel de compromiso deportivo: grado de diversión (4 ítemes), alternativas de implicación (4 ítemes), inversiones personales (3 ítemes), oportunidades de implicación (4 ítemes), coacciones sociales ( 7 ítemes) y compromiso deportivo (6 ítemes). Las respuestas fueron dadas a mediante una escala tipo likert con un rango de 1 a 5 , expresando nivel de acuerdo o desacuerdo con las afirmaciones.

\section{Análisis de la Información}

Para el análisis de los datos se utilizó SPSS 15.0.

Para comprobar si los datos tenían una distribución conocida, en este caso la distribución normal, se aplicaron las pruebas de K-S (Kolmogorov-Smirnova ${ }^{\mathrm{a}}$ ) y S-W (Shapiro-Wilk).

\section{Resultados}

A partir del análisis descriptivo de los datos se observa un alto nivel de compromiso deportivo frente a la actividad, el que se fundamenta en la relación existente entre los valores obtenidos por los distintos factores.

Para el factor grado de diversión, los datos se concentraron mayoritariamente en torno a la respuesta totalmente de acuerdo (valor 5), con una frecuencia de 76 sujetos, lo que corresponde al 56,3\% de las valoraciones totales para este factor. En la muestra total, para este factor, se observó una media de 4,70 con una desviación típica de 0,454 (Tabla 1 y Gráfico 1).

Tabla 1. Grados de Diversión.

\begin{tabular}{cccccc}
\hline & Frecuencia & Porcentaje & Porcentaje válido & Porcentaje acumulado \\
\hline 3,25 & 3 & 2,2 & 2,2 & 2,2 \\
& 3,50 & 3 & 2,2 & 2,2 & 4,4 \\
Válidos & 3 & 2,2 & 2,2 & 6,7 \\
& 3,75 & 8,1 & 8,1 & 14,8 \\
& 4,00 & 5,9 & 5,9 & 20,7 \\
& 4,25 & 11 & 8,1 & 8,1 & 28,9 \\
& 4,50 & 20 & 14,8 & 14,8 & 43,7 \\
& 4,75 & 56,3 & 56,3 & 100,0 \\
\hline
\end{tabular}


Gráfico 1. Grado de Diversión.

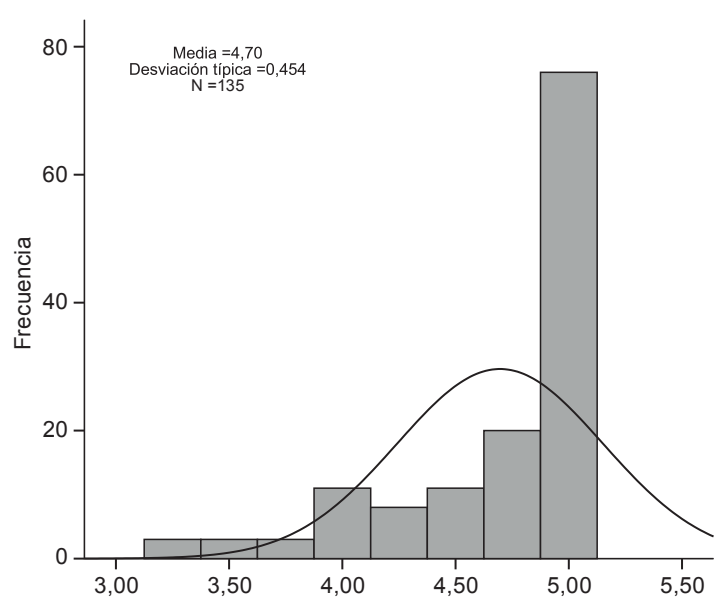

Gráfico 2. Inversiones Personales.

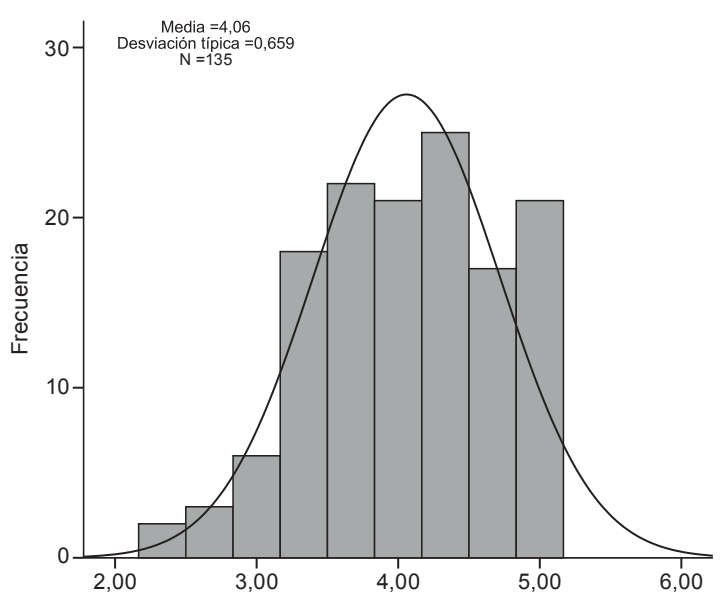

Tabla 2. Inversiones Personales.

\begin{tabular}{cccccc}
\hline & Frecuencia & Porcentaje & Porcentaje válido & Porcentaje acumulado \\
\hline \multirow{6}{*}{ Válidos } & & & 1,5 & 1,5 & 1,5 \\
& 2,33 & 3 & 2,2 & 2,2 & 3,7 \\
& 2,67 & 6 & 4,4 & 4,4 & 8,1 \\
& 3,00 & 13 & 13,3 & 13,3 & 37,5 \\
& 3,33 & 22 & 16,3 & 16,3 & 53,3 \\
& 3,67 & 21 & 15,6 & 15,6 & 71,9 \\
& 4,00 & 25 & 18,5 & 18,5 & 84,4 \\
& 4,33 & 17 & 12,6 & 12,6 & 100,0 \\
& 4,67 & 21 & 15,6 & 15,6 & \\
\hline
\end{tabular}

Con respecto al factor inversiones personales, las respuestas aparecen en mayor porcentaje en el segmento medio alto con una frecuencia de valores en torno a 4 , lo que representa al 53,3\%. La media correspondió a 4,06 y la desviación típica es 0,659 (Tabla 2 y Gráfico 2).

Frente a la variable oportunidades de implicación, los resultados arrojados se sitúan en valores altos, situándose el $43,7 \%$ de los casos en las puntuaciones superiores $(4,75$ y 5$)$, con una frecuencia de 27 y 32 casos respectivamente. En este factor, la media correspondió a 4,37 y la desviación típica es de 0,595 (Tabla 3 y Gráfico 3).

En relación a esta variable, se observan altos niveles, situándose el 43,7\% en los más altos, lo que quiere decir que los participantes conciben que les han dado más oportunidades de involucramiento, como resultado de la participación continua.

El cuarto factor relacionado al Compromiso Deportivo, coacciones sociales, mostró una mayor amplitud en sus respuestas, lo que se refleja al observar la existencia de una similar distribución de datos tanto en los valores altos como en los bajos. Esto se puede observar en la presencia de frecuencias altas y bajas en ambos extremos de la tabla. La media se ubicó en el centro exacto de la escala de evaluación $(2,50)$ y la desviación típica en 0,926 (Tabla 4 y Gráfico 4).

Frente a esta variable, los datos se encuentran más dispersos, no obstante, la media muestra valores medios de coacción social, es decir, las expectativas y normas sociales están influyendo de forma moderada 
Gráfico 3. Oportunidades de Implicación.

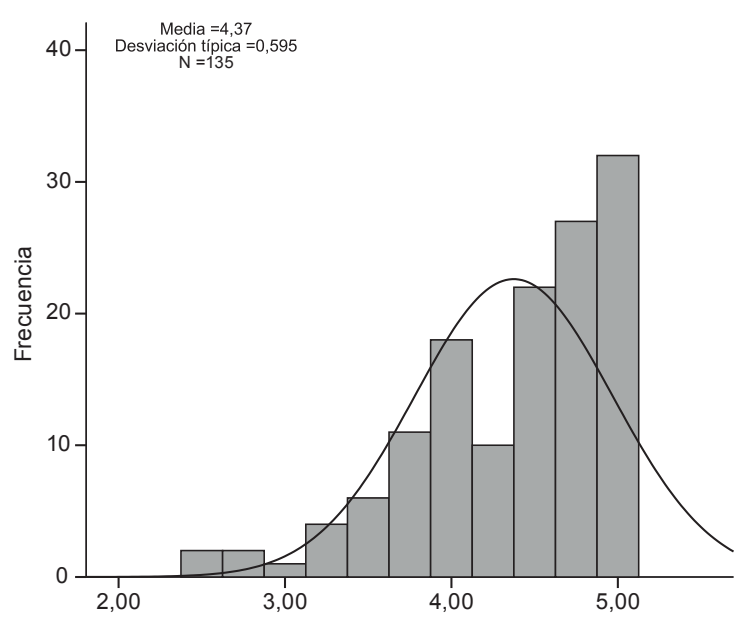

Gráfico 4. Coacciones Sociales.

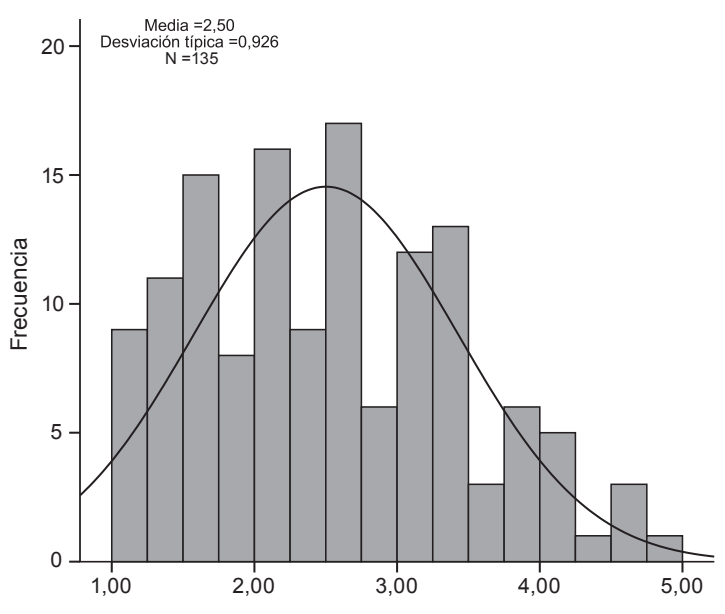

Tabla 3. Oportunidades de Implicación.

\begin{tabular}{cccccc}
\hline & Frecuencia & Porcentaje & Porcentaje válido & Porcentaje acumulado \\
\hline \multirow{6}{*}{ Válidos } & 2 & 1,5 & 1,5 & 1,5 \\
& 2,50 & 2 & 1,5 & 1,5 & 3,0 \\
& 2,75 & 1 &, 7 &, 7 & 3,7 \\
& 3,00 & 4 & 3,0 & 3,0 & 6,7 \\
& 3,25 & 6 & 4,4 & 8,4 & 11,1 \\
& 3,50 & 11 & 8,1 & 13,3 & 3,3 \\
& 3,75 & 18 & 7,4 & 7,4 & 40,0 \\
& 4,00 & 10 & 16,3 & 16,3 & 56,3 \\
& 4,25 & 22 & 20,0 & 20,0 & 76,3 \\
& 4,50 & 27 & 23,7 & 23,7 & 100,0 \\
\hline
\end{tabular}

en el compromiso deportivo. Estas expectativas se expresan en sentimientos de obligatoriedad con respecto a la práctica.

Las alternativas de implicación, como la quinta variable en el Compromiso Deportivo, muestra que la mayoría de los datos se ubica en el segmento medio bajo $(64,4 \%)$, con una frecuencia de 30 respuestas para el valor 2. En este caso la media fue de 2,43 y la desviación típica de 0,956 (Tabla 5 y Gráfico 5).

Con respecto a este factor, si bien se observan datos dispersos, un $22 \%$ se concentra en un valor medio bajo, lo que indica que un quinto de la muestra considera que el resto de actividades que podría realizar son poco atractivas en compración con su deporte, sin embargo, considerando la media se puede decir que esta variable se presenta a niveles moderados o medio.

Finalmente, frente al último factor evaluado en el instrumento SCQ-e, se puede observar un alto nivel de compromiso deportivo por parte de la muestra hacia la actividad, es decir, tienen la intención de seguir participando.

La mayor frecuencia de valores $(n=41)$, correspondiente al $30,4 \%$, se ubica en la respuesta 5 , lo que representa la puntuación máxima. Igualmente los valores de puntuación inmediatamente anterior que se encuentran en el segmento superior de valoración, reflejan el alto compromiso deportivo cubriendo $38,5 \%$ del porcentaje total. La media se ubicó en el valor 4,56 y la desviación típica en 0,451 (Tabla 6 y Gráfico 6). 
Tabla 4. Coacciones Sociales.

\begin{tabular}{|c|c|c|c|c|c|}
\hline & & Frecuencia & Porcentaje & Porcentaje válido & Porcentaje acumulado \\
\hline & 1,00 & 6 & 4,4 & 4,4 & 4,4 \\
\hline & 1,14 & 3 & 2,2 & 2,2 & 6,7 \\
\hline & 1,29 & 7 & 5,2 & 5,2 & 11,9 \\
\hline & 1,43 & 4 & 3,0 & 3,0 & 14,8 \\
\hline & 1,57 & 7 & 5,2 & 5,2 & 20,0 \\
\hline & 1,71 & 8 & 5,9 & 5,9 & 25,9 \\
\hline & 1,86 & 8 & 5,9 & 5,9 & 31,9 \\
\hline & 2,00 & 6 & 4,4 & 4,4 & 36,3 \\
\hline & 2,14 & 10 & 7,4 & 7,4 & 43,7 \\
\hline & 2,29 & 5 & 3,7 & 3,7 & 47,4 \\
\hline & 2,43 & 4 & 3,0 & 3,0 & 50,4 \\
\hline & 2,50 & 1 &, 7 &, 7 & 51,1 \\
\hline & 2,57 & 9 & 6,7 & 6,7 & 57,8 \\
\hline & 2,71 & 7 & 5,2 & 5,2 & 63,0 \\
\hline \multirow[t]{15}{*}{ Válidos } & 2,86 & 6 & 4,4 & 4,4 & 67,4 \\
\hline & 3,00 & 4 & 3,0 & 3,0 & 70,4 \\
\hline & 3,14 & 8 & 5,9 & 5,9 & 76,3 \\
\hline & 3,29 & 8 & 5,9 & 5,9 & 82,2 \\
\hline & 3,43 & 5 & 3,7 & 3,7 & 85,9 \\
\hline & 3,50 & 1 &, 7 &, 7 & 86,7 \\
\hline & 3,57 & 2 & 1,5 & 1,5 & 88,1 \\
\hline & 3,86 & 6 & 4,4 & 4,4 & 92,6 \\
\hline & 4,00 & 4 & 3,0 & 3,0 & 95,6 \\
\hline & 4,14 & 1 &, 7 &, 7 & 96,3 \\
\hline & 4,29 & 1 &, 7 &, 7 & 97,0 \\
\hline & 4,57 & 1 &, 7 &, 7 & 97,8 \\
\hline & 4,71 & 2 & 1,5 & 1,5 & 99,3 \\
\hline & 4,86 & 1 &, 7 & ,7 & 100,0 \\
\hline & Total & 135 & 100,0 & 100,0 & \\
\hline
\end{tabular}

Tabla 5. Alternativas de Implicación.

\begin{tabular}{|c|c|c|c|c|c|}
\hline & & Frecuencia & Porcentaje & Porcentaje válido & Porcentaje acumulado \\
\hline \multirow{19}{*}{ Válidos } & 1,00 & 15 & 11,1 & 11,1 & 11,1 \\
\hline & 1,25 & 3 & 2,2 & 2,2 & 13,3 \\
\hline & 1,50 & 4 & 3,0 & 3,0 & 16,3 \\
\hline & 1,75 & 10 & 7,4 & 7,4 & 23,7 \\
\hline & 2,00 & 30 & 22,2 & 22,2 & 45,9 \\
\hline & 2,25 & 12 & 8,9 & 8,9 & 54,8 \\
\hline & 2,33 & 1 &, 7 &, 7 & 55,6 \\
\hline & 2,50 & 12 & 8,9 & 8,9 & 64,4 \\
\hline & 2,75 & 13 & 9,6 & 9,6 & 74,1 \\
\hline & 3,00 & 6 & 4,4 & 4,4 & 78,5 \\
\hline & 3,25 & 8 & 5,9 & 5,9 & 84,4 \\
\hline & 3,50 & 2 & 1,5 & 1,5 & 85,9 \\
\hline & 3,75 & 3 & 2,2 & 2,2 & 88,1 \\
\hline & 4,00 & 6 & 4,4 & 4,4 & 92,6 \\
\hline & 4,25 & 5 & 3,7 & 3,7 & 96,3 \\
\hline & 4,50 & 2 & 1,5 & 1,5 & 97,8 \\
\hline & 4,75 & 2 & 1,5 & 1,5 & 99,3 \\
\hline & 5,00 & 1 &, 7 &, 7 & 100,0 \\
\hline & Total & 135 & 100,0 & 100,0 & \\
\hline
\end{tabular}


Gráfico 5. Alternativas de Implicación.

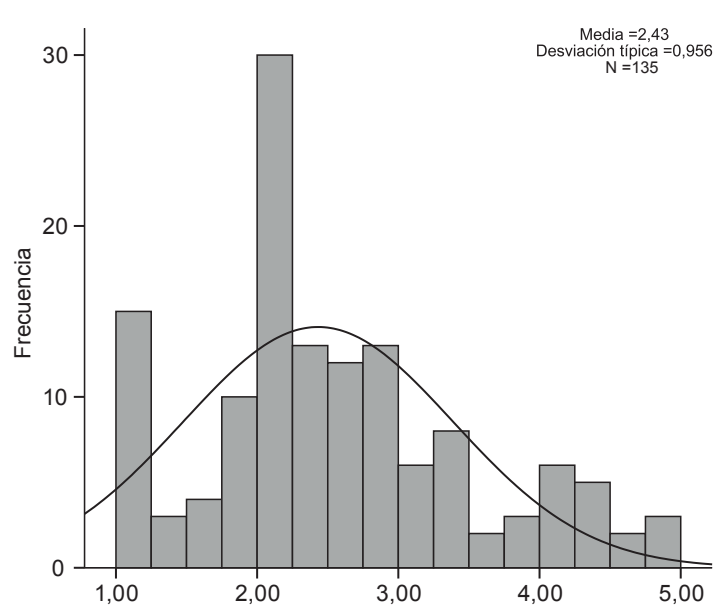

Gráfico 6. Compromiso Deportivo.

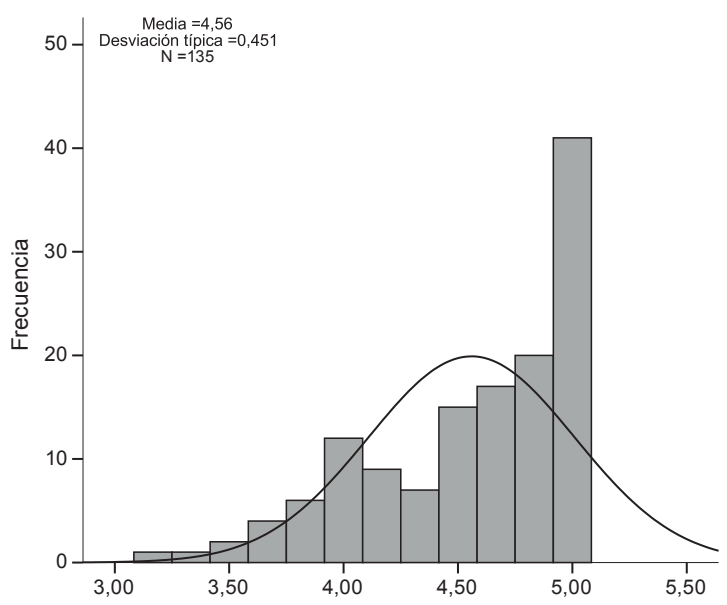

Tabla 6. Compromiso Deportivo.

\begin{tabular}{|c|c|c|c|c|c|}
\hline & & Frecuencia & Porcentaje & Porcentaje válido & Porcentaje acumulado \\
\hline \multirow{13}{*}{ Válidos } & 3,17 & 1 &, 7 &, 7 &, 7 \\
\hline & 3,33 & 1 &, 7 & ,7 & 1,5 \\
\hline & 3,50 & 2 & 1,5 & 1,5 & 3,0 \\
\hline & 3,67 & 4 & 3,0 & 3,0 & 5,9 \\
\hline & 3,83 & 6 & 4,4 & 4,4 & 10,4 \\
\hline & 4,00 & 12 & 8,9 & 8,9 & 19,3 \\
\hline & 4,17 & 9 & 6,7 & 6,7 & 25,9 \\
\hline & 4,33 & 7 & 5,2 & 5,2 & 31,1 \\
\hline & 4,50 & 15 & 11,1 & 11,1 & 42,2 \\
\hline & 4,67 & 17 & 12,6 & 12,6 & 54,8 \\
\hline & 4,83 & 20 & 14,8 & 14,8 & 69,6 \\
\hline & 5,00 & 41 & 30,4 & 30,4 & 100,0 \\
\hline & Total & 135 & 100,0 & 100,0 & \\
\hline
\end{tabular}

\section{Discusión}

Considerando que los resultados obtenidos reafirman lo señalado por otros autores, se concuerda sobre el hecho de que ciertos factores se ajustan positivamente con el Compromiso Deportivo (Grado de disfrute, inversiones personales y oportunidades de implicación). En el caso de las coacciones sociales se coincide con otras investigaciones en cuanto a que no queda claro su ajuste con el Compromiso Deportivo. Existen trabajos que han obtenido resultados que no corroboran todas las predicciones del modelo teórico (Carpenter \& Scanlan, 1998; Scanlan, Russel, Beals \& Scanlan, 2003), sin embargo, en otros estudios las coacciones sociales predicen positivamente el compromiso aunque de manera débil, presentando cierto grado de coherencia con lo postulado en el modelo teórico (Alexandris, Zahariadis, Tsorbatzoudis \& Grouios, 2002; Weiss, Kimmel \& Smith, 2001; Weiss \& Weiss, 2007).

En este caso se constata que la muestra presenta un alto nivel de Compromiso Deportivo y analizando cada uno de los factores se observa que hay concordancia en que el compromiso deportivo, oportunidades de implicación e inversiones personales tienen un efecto positivo con el Compromiso Deportivo, mientras que queda clara la relación entre alternativas de implicación y Compromiso Deportivo. La relación incierta se da entre Compromiso Deportivo y Coacciones sociales, ya que los datos se encuentran repartidos. Esto concuerda con hallazgos presentados en otros estudios (García, Leo, Martín \& Sánchez, 2008; Souza, 2008). 


\section{Conclusiones}

Existe un alto nivel de compromiso deportivo, concordando con la teoría de Compromiso Deportivo en cuanto a la relación positiva y negativa existentes entre las variables que se evalúan en el instrumento y el nivel total de compromiso deportivo para con la actividad (Souza, C., Torregrosa, M., Viladrich, C., Villamarín, F. \& Cruz, J., 2007). Es decir, se puede concluir que los deportistas evaluados poseen una disposición psicológica positiva, que se refleja en el deseo y decisión de seguir participando en su respectiva disciplina deportiva. Este resultado se transforma en un factor protector frente al abandono deportivo y como agente facilitador de la permanencia en la actividad.

Desde la perspectiva de la motivación de logro, el alto grado de compromiso deportivo otorgado por los resultados en grado de diversión, inversiones personales y oportunidades de implicación, se relacionan positivamente con el desarrollo de una orientación motivacional a la tarea, la cual, en las edades estudiadas, otorga la base motivacional que potencia la adherencia y evita el abandono, en cuanto valora el esfuerzo personal y la diversión como elementos claves cuando se evalúa la participación y la consecución del éxito deportivo, siendo, por tanto, información útil a la hora de trabajar elementos psicológicos que coadyuve el entrenamiento deportivo.

\section{Referencias}

Alexandris, K., Zahariadis, P., Tsorbatzoudis, C. \& Grouios, G. (2002). Testing the sport commitment model in the context of exercise and fitness participation. Journal of Sport Behavior, 25 (3), 217- 230

Carpenter, P. \& Scanlan, T. (1993). A test of the sport commitment model using structural equation modeling. Journal of Sport and exercise Psychology, 15, 119-133.

Cecchini, J. A., Méndez, A., \& Muñiz, J. (2002). Motives for practicing sport in Spanish schoolchildren. Psicothema, 14, 523-531.
Cervelló, E., Hutzler, Y., Reina, R., Sanz, D., \& Moreno, J. A. (2005). Goal orientations, contextual and situational motivational climate and competition goal involvement in Spanish athletes with cerebral palsy. Psicothema, 17, 633-638.

García Más, A., Palou, P., Gili, M., Ponseti, X., Borrás, P., Vidal, J., Cruz, J., Torregrosa, M., Villamarín, F. \& Sousa, C. (2010). Selfdetermination and Sport Commitment in Young Soccer Competitive Player. Spanish Journal of Psychology (Aceptado en prensa).

García,T., Leo, F., Martín, E. \& Sánchez, P. (2008). El Compromiso Deportivo y su relación con factores disposicionales y situacionales contextuales de la motivación. International Journal of Sport Science, 12(4), 45-58.

Kelley, H. \& Thibaut, J. (1978). Interpersonal relations: A theory of independence. New York: Wiley- Interscience.

Morales, V., Hernández-Mendo, A., \& Blanco, A. (2005). Evaluación de la calidad en los programas de actividad física. Psicothema, 17, 311-317.

Rusbult, C. (1980). Commitment and satisfaction in romantic associations: A test of the investment model. Journal of Experimental Social Psychology, 16, 172-186.

Rusbult, C. E. \& Farrell, D. (1983). A longitudinal test of the investment model: The impact on job satisfaction, job commitment and turnover of variations in rewards, costs, alternatives and investments. Journal of Applied Psychology, 68, 429-438.

Scanlan, T. K., Carpenter, P. J., Schmidt, G. W., Simons, J. P. \& Keeler, B. (1993). An Introduction to the Sport Commitment Model. Journal of Sport \& Exercise Psychology, 15(1), 1-15.

Scanlan, T., Russel, D., Beals, K. \& Scanlan, L. (2003). Project on elite athlete commitment (Peak): II. A direct test and expansion of the sport commitment model with elite amateur sportsmen. Journal of Sport and Exercise Psychology, 25, 377-401.

Souza, C. (2008). Efectos del Programa de Asesoramiento Personalizado a Entrenadores (PAPE) en la conducta y en el Compromiso Deportivo. Tesis Doctoral. Departamento de Psicología Básica, Evolutiva i de L' Educació. Universitat Autónoma de Barcelona.

Souza, C., Torregrosa, M., Viladrich, C., Villamarín, F. \& Cruz, J. (2007). The Commitment of Young Soccer Players. Psicothema, $19,2,256-262$

Thomas, Jerry R. \& Nelson, Jack K. (2007). Métodos de investigación en actividad física. Barcelona: Paidotribo.

Weiss, M. \& Petlichkoff, L. (1989). Children's motivation for participation in withdrawal from sport: Identifying the missing links. Pediatric Exercise Science, 1, 195-211.

Weiss, M., Kimmel, L. \& Smith, A. (2001). Determinants of Sport Commitment among junior tennis players: Enjoyment as a mediating variables. Pediatric Exercise Science, 13, 131-144. 